\title{
ROLAMENTOS AUTOCOMPENSADORES DE ROLOS COM MICROSTRUTURA BAINÍTICA VS MARTENSÍTICA EM MÁQUINA DE LINGOTAMENTO CONTÍNUO*
}

Fabricio Sartori Trani ${ }^{1}$

\section{Resumo}

Os rolamentos da máquina de lingotamento contínuo de placas foram inspecionados após campanha do segmento. O tempo de operação foi de uma campanha (1,5 ano ou 1,5 milhão de toneladas produzidas). Nos mancais centrais dos rolos longos, foram inspecionados 14 rolamentos, sendo 2 deles SKF com microestrutura bainítica e os demais 12 de outro fabricante, com microestrutura martensítica. Os rolamentos SKF eram de classe de desempenho Explorer e aprimorados, ficando evidenciado modo de falha seguro, diferentemente dos demais rolamentos, que apresentaram fadiga subsuperficial acentuada (descascamento), fraturas por fadiga e superaquecimento. A aplicação de lingotamento contínuo é bastante crítica para rolamentos, pois contempla sobrecarga, cargas de choque, contaminação por água e particulado sólido e altas temperaturas de operação. Ainda assim, a vida em serviço dos rolamentos com microestrutura bainítica foi estendida.

Palavras-chave: Rolamentos; Microestrutura; Vida; Lingotamento.

\section{SPHERICAL ROLLER BEARINGS BAINITIC MICROSTRUCTURE VS MARTENSITIC MICROSTRUCTURE IN CONTINUOUS CASTING MACHINE}

\begin{abstract}
The bearings of the slab continuous casting machine were inspected after the campaign of a segment. The operation time was one campaign (1,5 year or 1,5 million of tonnes produced). In the central bearings of the long rolls, 14 roller bearings were inspected, being 2 of these SKF with bainitic microstructure and the other 12 of another manufacturer with martensitic microstructure. The SKF bearings were at Explorer performance class and upgraded, evidencing safe failure mode, differently from the other bearings, which presented strong subsurface initiated fatigue (spalling), fatigue fractures and overheating. The continuous casting application is very critical for bearings, as it contemplates overloading, shock loadings, water and solid contamination and high operation temperatures. Even though, bainitic microstructe bearings service life was extended.
\end{abstract}

Keywords: Bearings; Microstructure; Life; Casting. 


\section{INTRODUÇÃO}

Os 14 rolamentos avaliados neste estudo operaram nos mancais centrais (lado fixo) dos rolos longos da máquina de lingotamento contínuo de placas. A cada posição no segmento, o lado de montagem dos rolos curtos e longos é invertido, de forma que a placa não fique marcada pelo gap entre os rolos. Foram analisadas somente as pistas dos anéis externos, pois não havia tempo hábil para desmontar os rolamentos e analisar também as pistas dos anéis internos devido ao volume grande de peças a serem inspecionadas. A Figura 1 destaca o rolamento da posição central do rolo longo.

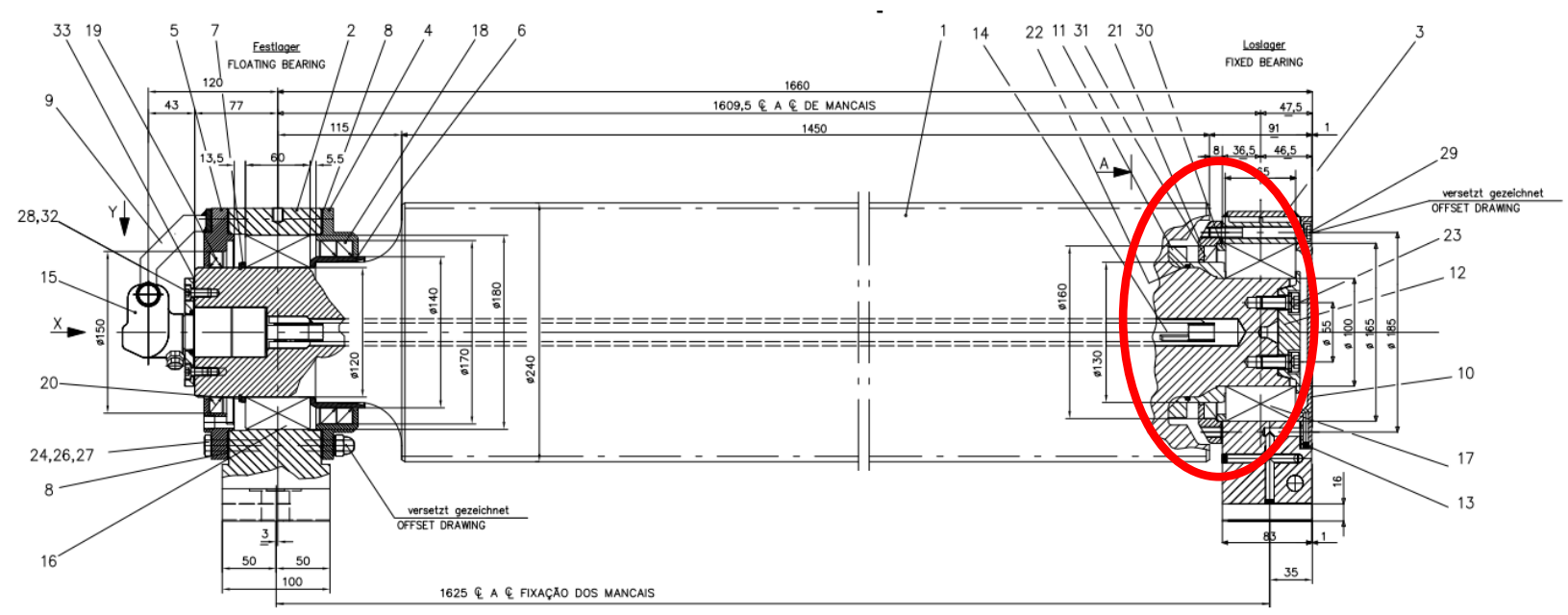

Figura 1. Desenho do rolo longo.

\subsection{Designação dos Rolamentos}

Os 2 rolamentos SKF com microestrutura bainítica que operaram na posição central dos rolos longos são de designação 24120 CC/C3W33, conforme destacado na Figura 2. A mesma figura destaca também que os rolamentos estão na classe de desempenho Explorer e são aprimorados (WR).

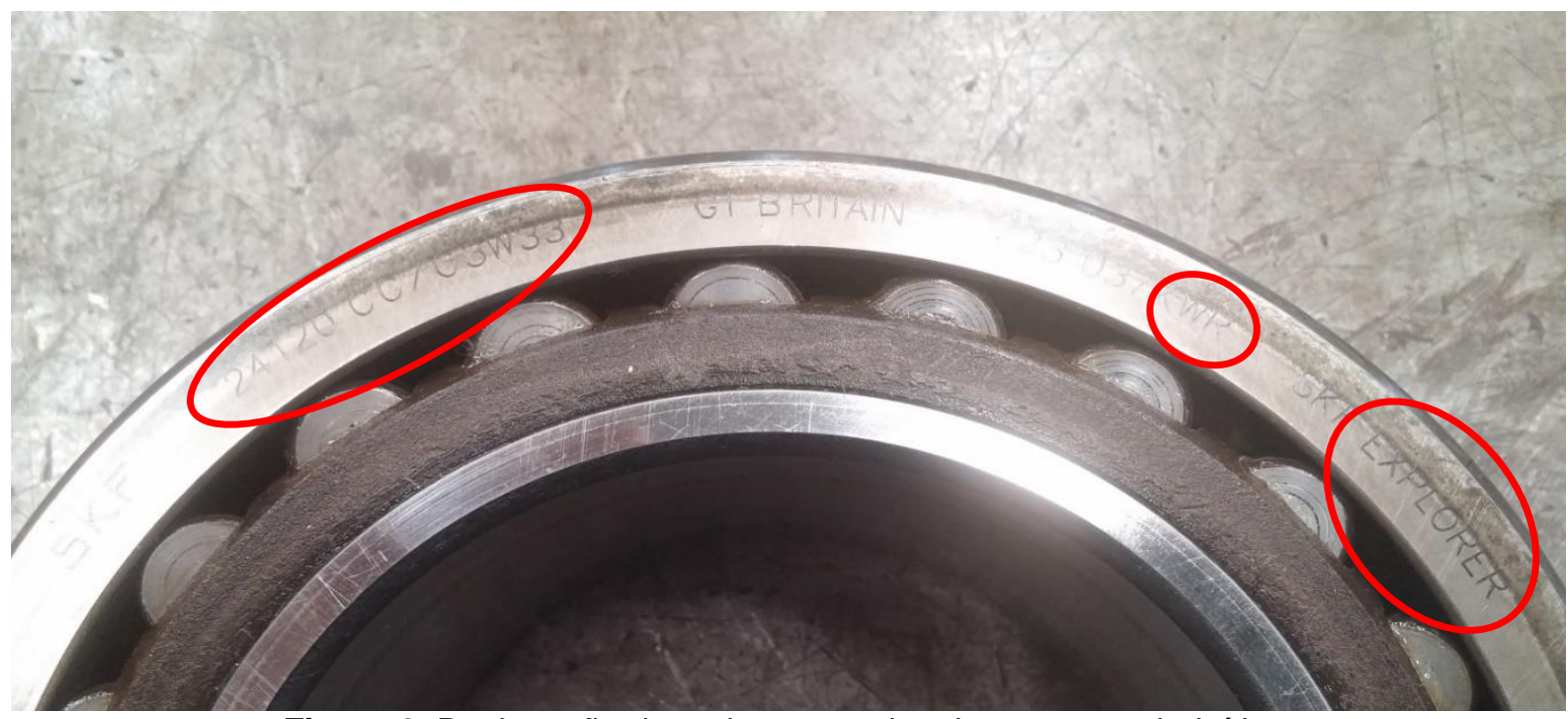

Figura 2. Designação dos rolamentos de microestrutura bainítica. 


\subsection{Objetivo}

O objetivo deste trabalho é apresentar estudo comparativo de desempenho de rolamentos com microestrutura bainítica e martensítica, com base nos modos de falha apresentados em campo, classificados de acordo com a norma ISO 15243. Em adição, serão apresentados em detalhe todos os benefícios dos rolamentos SKF Explorer aprimorados, bem como a influência das melhorias combinadas na vida do rolamento.

\section{MATERIAIS E MÉTODOS}

\subsection{Dados da Aplicação}

Os dados da aplicação constam na Tabela 1:

Tabela 1. Dados da aplicação.

\begin{tabular}{|l|l|}
\hline Aplicação & $\begin{array}{l}\text { Equipamento: Máquina de lingotamento contínuo } \\
\text { Processo: Lingotamento de placas }\end{array}$ \\
\hline Rolamentos & $\begin{array}{l}\text { Fabricante: SKF / outro } \\
\text { Quantidade: } 2 \text { / 12 }\end{array}$ \\
\hline Ambiente & Severo (impurezas, umidade, cargas de choque) \\
\hline \multirow{3}{*}{ Lubrificação } & $\begin{array}{l}\text { Tipo: Graxa } \\
\text { Método: Centralizado/automático } \\
\text { Designação: Shell Gadus S3 V460 } \\
\text { Vazão: } 3,97 \text { I/min }\end{array}$ \\
\hline Cargas & Intensidade pesada, valores não informados \\
\hline Rotação & 1 rpm \\
\hline Temperatura & $\begin{array}{l}\text { Média: } 90^{\circ} \mathrm{C} \\
\text { Picos: } 140^{\circ} \mathrm{C}\end{array}$ \\
\hline Vedações & Retentor radial e anel-O \\
\hline Ajustes & Eixo: e8 \\
Caixa: G7
\end{tabular}

\subsection{Modos de Falha}

A Figura 3 evidencia modo de falha seguro em um dos rolamentos com microestrutura bainítica, com marcas de trabalho e desgaste abrasivo em estágio inicial. Foi constatado alto índice de contaminação por água, que é inerente à aplicação devido à refrigeração dos mancais, gerando corrosão por umidade na pista do anel externo. 


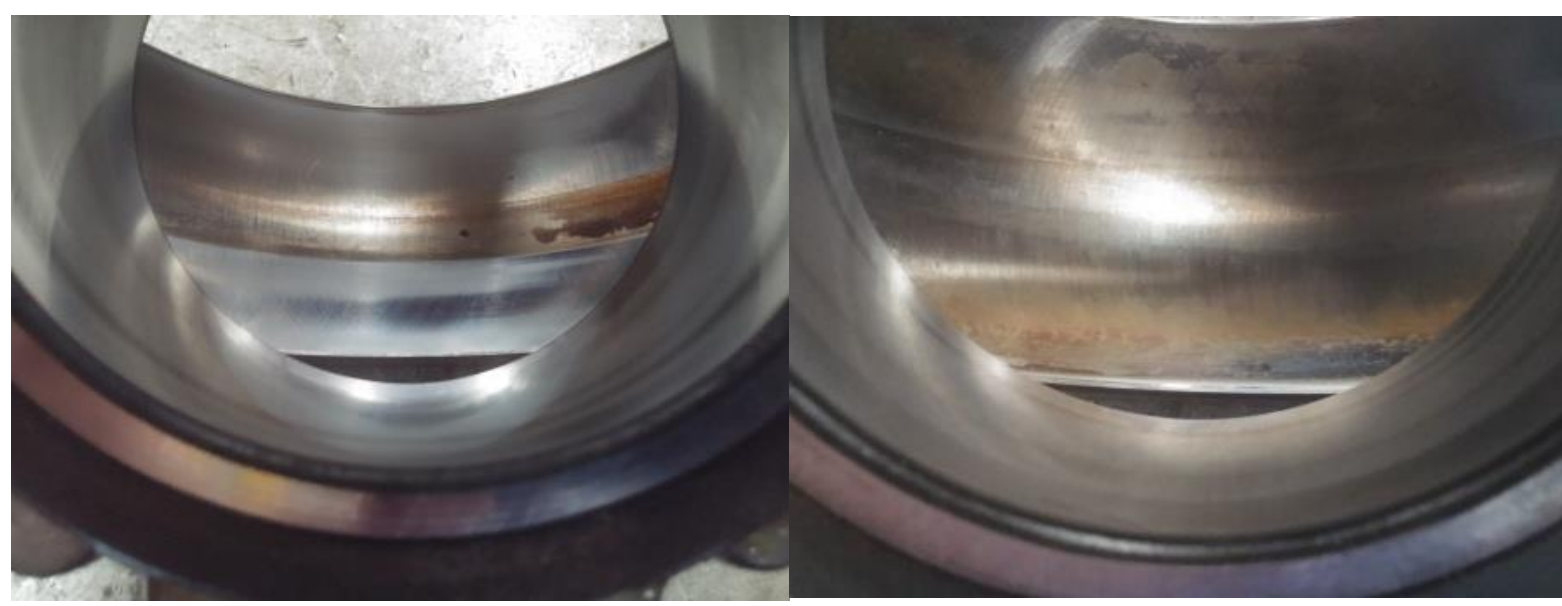

Figura 3. Modo de falha seguro em um rolamento com microestrutura bainítica.

A Figura 4 evidencia modo de falha seguro no outro rolamento com microestrutura bainítica.

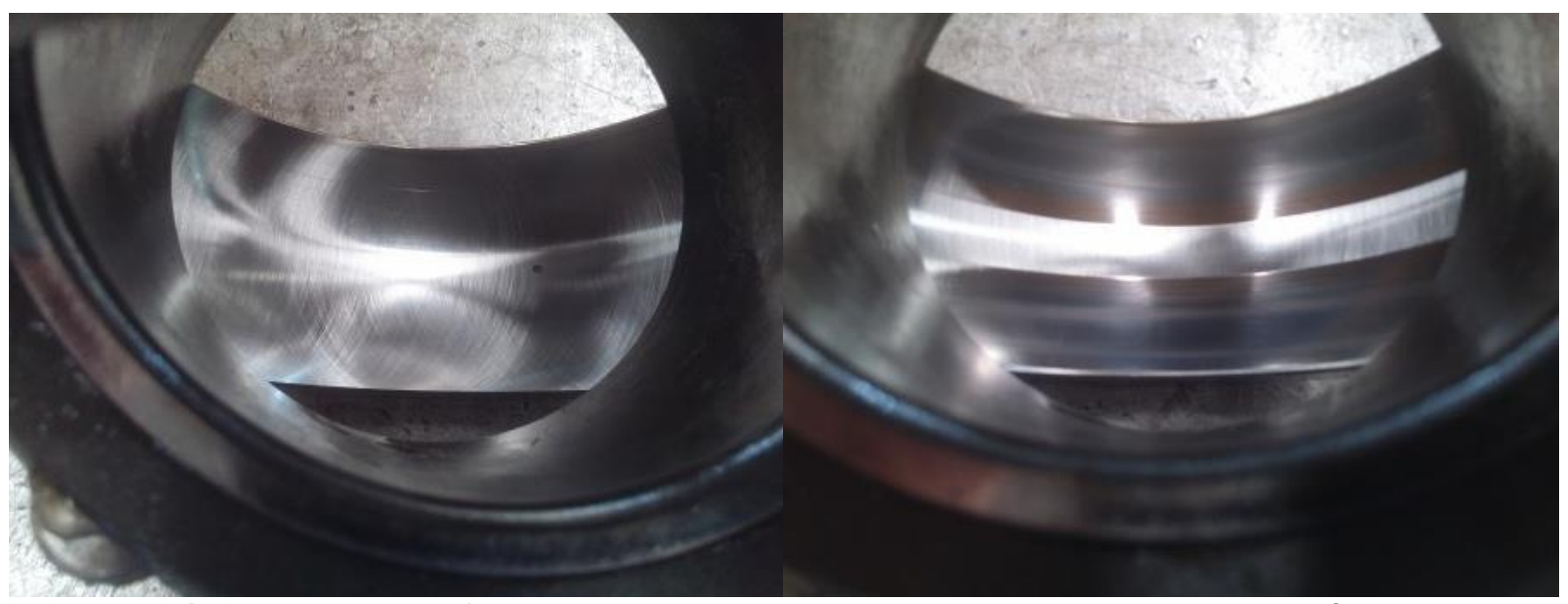

Figura 4. Modo de falha seguro no outro rolamento com microestrutura bainítica.

A Figura 5 evidencia modos de falha críticos, classificados de acordo com a norma ISO 15243, como fadiga subsuperficial e fraturas por fadiga, presenciados nos 12 rolamentos com microestrutura martensítica, que possuem a mesma designação principal e operaram no mesmo segmento e campanha dos 2 rolamentos com microestrutura bainítica. 


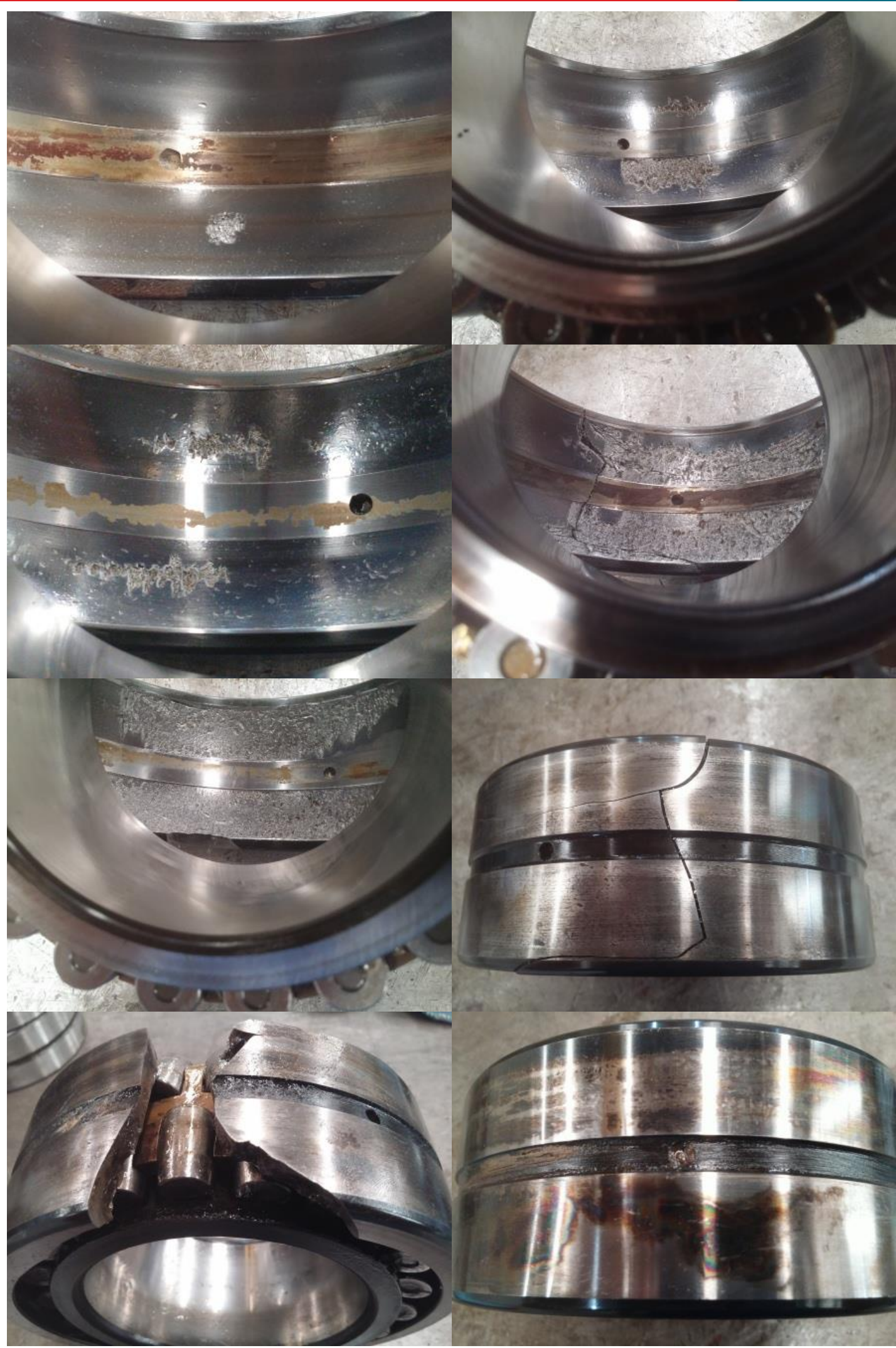

Figura 5. Modos de falha críticos em rolamentos com microestrutura martensítica. 


\section{RESULTADOS E DISCUSSÃO}

\subsection{Características e Benefícios dos Rolamentos SKF com Microestrutura Bainítica}

Os rolamentos autocompensadores de rolos SKF Explorer aprimorados com microestrutura bainítica apresentam uma série de melhorias, tais como:

- Aço ultra limpo e homogêneo, com maior índice de pureza, não permitindo a geração de picos de tensão sob carga;

- Tratamento térmico diferenciado, fornecendo maior dureza e resistência ao desgaste aos rolamentos, sem perda de tenacidade e com a microestrutura bainítica aprimorada;

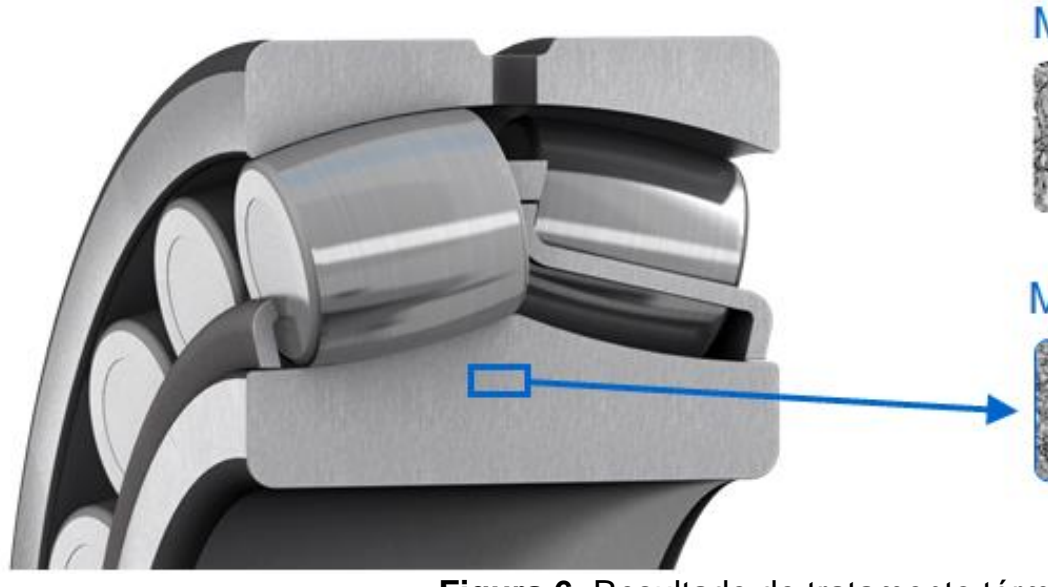

\section{Microestrutura anterior}

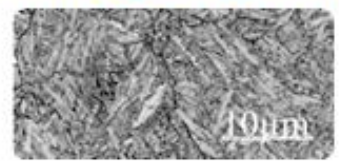

Microestrutura aprimorada

Figura 6. Resultado do tratamento térmico.

- Geometria interna dos componentes otimizada, provendo melhor distribuição de tensões, e anel-guia flutuante para controlar os rolos não-carregados e mantê-los na posição ideal, minimizando atrito e geração de calor;

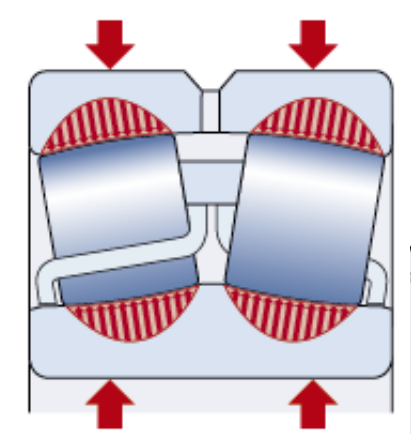

(a)

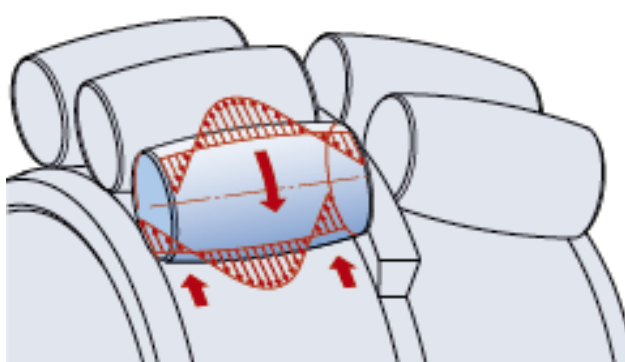

(b)

Figura 7. Distribuição de carga (a) e distribuição de calor por atrito (b).

- Acabamento superficial aprimorado, melhorando as superfícies de contato de todos os componentes, permitindo a minimização dos efeitos de atrito e a maximização do efeito do lubrificante;

- Maior precisão de giro (P5 como padrão) e tolerâncias de largura mais apertadas para diâmetros de furo de até $300 \mathrm{~mm}$. 


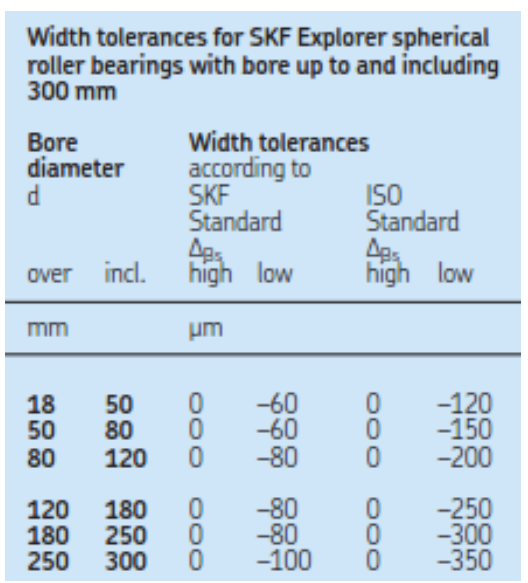

Figura 8. Tolerâncias de largura

Todas estas melhorias combinadas fornecem ao rolamento e aplicação os seguintes benefícios:

- Maior capacidade de carga dinâmica em comparação a rolamentos de projeto convencional;

- Maior resistência ao desgaste e à contaminação;

- Menor temperatura de operação e maior vida do lubrificante;

- Redução dos níveis de ruído e vibração;

- Excelente desempenho em altas velocidades;

- Significativo aumento da vida em serviço, principalmente em ambientes com altos índices de contaminação e/ou lubrificação deficiente;

- Maior produtividade, confiabilidade e menor tempo de máquina parada.

\subsection{Influência das Melhorias na Vida do Rolamento}

Testes realizados nos laboratórios SKF mostram que os rolamentos autocompensadores de rolos SKF Explorer aprimorados com microestrutura bainítica podem durar até o dobro dos rolamentos anteriores em condições de lubrificação deficiente ou de contaminação, conforme Figura 9:

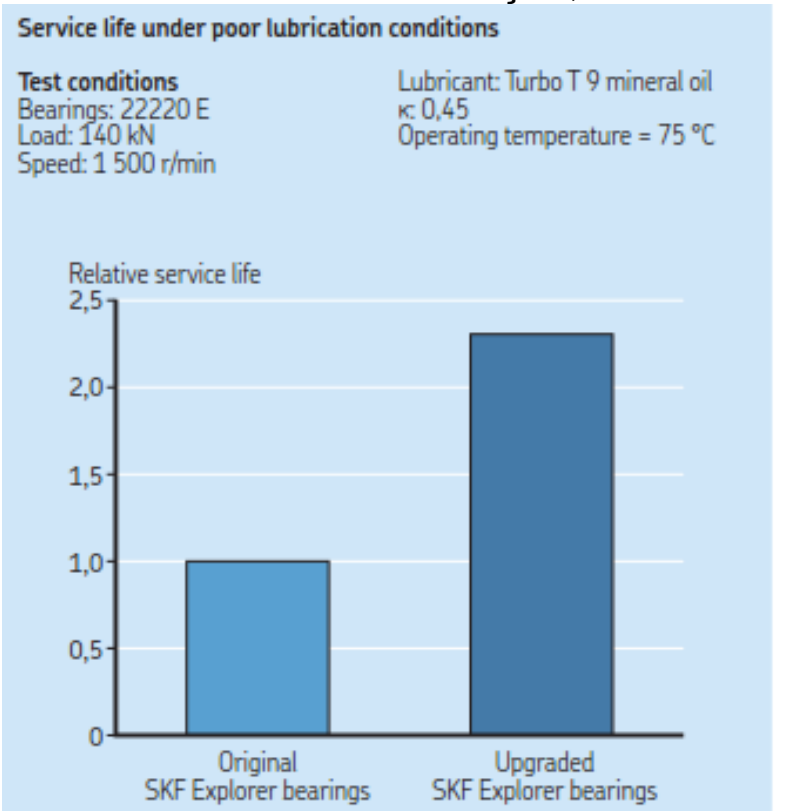

(a)
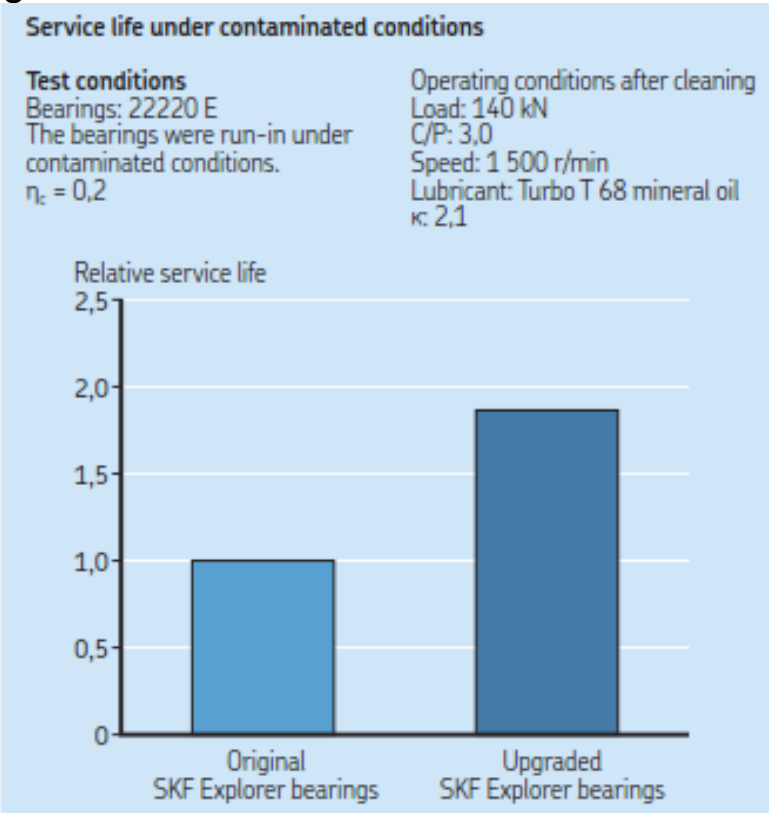

(b)

Figura 9. Vida em serviço dobrada sob condições de lubrificação deficiente (a) e contaminação (b). 
A Figura 10 traz comparativo da vida em serviço entre rolamentos com microestrutura bainítica e rolamentos de demais fabricantes, evidenciando os benefícios dos rolamentos SKF Explorer aprimorados:

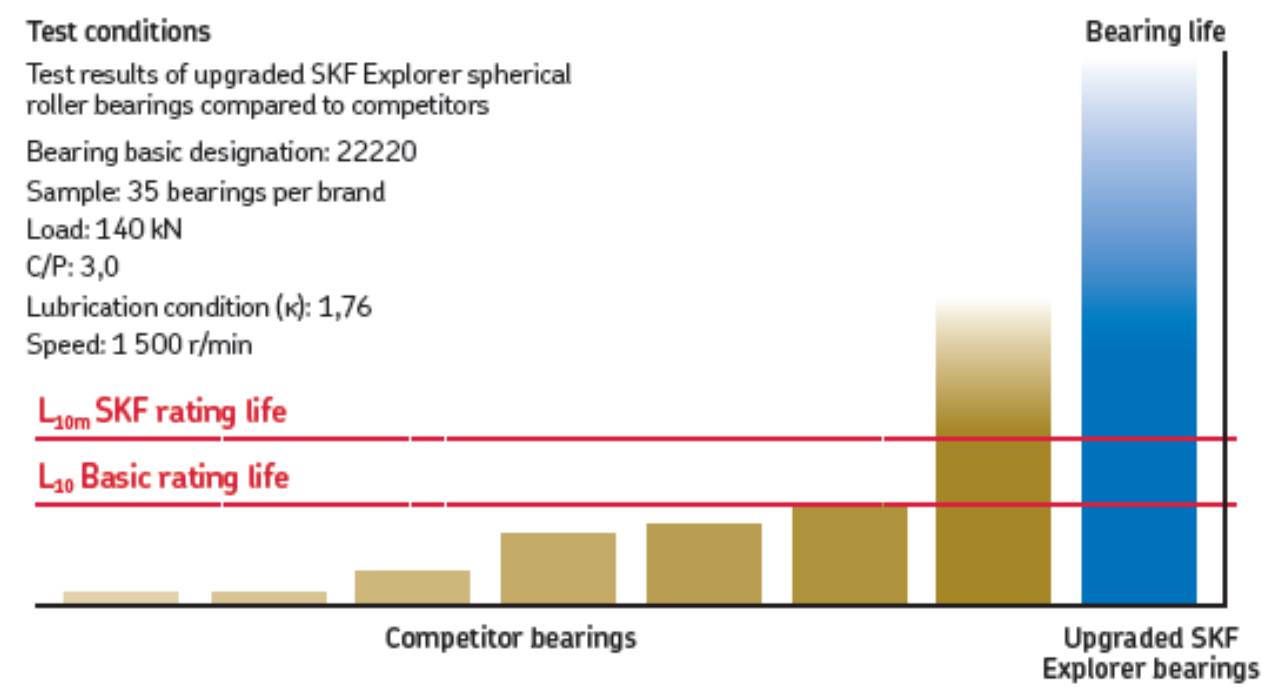

Figura 10. Vida em serviço de diferentes fabricantes de rolamentos.

O cálculo de vida à fadiga modificada foi introduzido pela SKF em 2003, com base na norma ISO 281, estabelecido pela equação 1:

Onde:

$$
L_{10 m}=a_{1} \times a_{S K F} \times\left(\frac{C}{P}\right)^{\frac{10}{3}} \times \frac{1000000}{60 \times n}
$$

$\mathrm{L}_{10 \mathrm{~m}}=$ Vida à fadiga modificada SKF, horas

$\mathrm{a}_{1}=$ fator de confiabilidade

askf $=$ fator de modificação de vida SKF

$\mathrm{C}$ = capacidade de carga dinâmica do rolamento, $\mathrm{kN}$

$\mathrm{P}=$ carga dinâmica equivalente do rolamento, $\mathrm{kN}$

$\mathrm{n}=$ rotação, $\mathrm{rpm}$

O fator de modificação de vida askf representa uma relação bastante complexa entre vários fatores, como carga limite de fadiga, contaminação e lubrificação. Os valores de carga limite de fadiga $\left(\mathrm{P}_{\mathrm{u}}\right)$ são fornecidos nas tabelas do catálogo geral. As condições de contaminação são dadas pelo fator de contaminação $\eta_{c}$. As condições de lubrificação são fornecidas pela equação 2 :

Onde:

$$
K=\frac{v}{v_{1}}
$$

$\mathrm{K}=$ fator de viscosidade (Kappa)

$v=$ viscosidade real do lubrificante à temperatura de operação

$\mathrm{V}_{1}$ = viscosidade requerida para o lubrificante à temperatura de operação, em função do diâmetro médio do rolamento e rotação

Os valores de askf podem ser obtidos pelo diagrama da Figura 11. Para rolamentos autocompensadores de rolos padrão, os valores das abscissas são mostrados em preto e, para rolamentos SKF Explorer, em azul. Na prática, para rolamentos SKF Explorer, foi estabelecido que se deve multiplicar $\eta_{c}(P u / P)$ por um fator de 1,4 como expressão dos refinamentos de extensão de vida destes rolamentos, o que representa exatamente os valores em azul. 


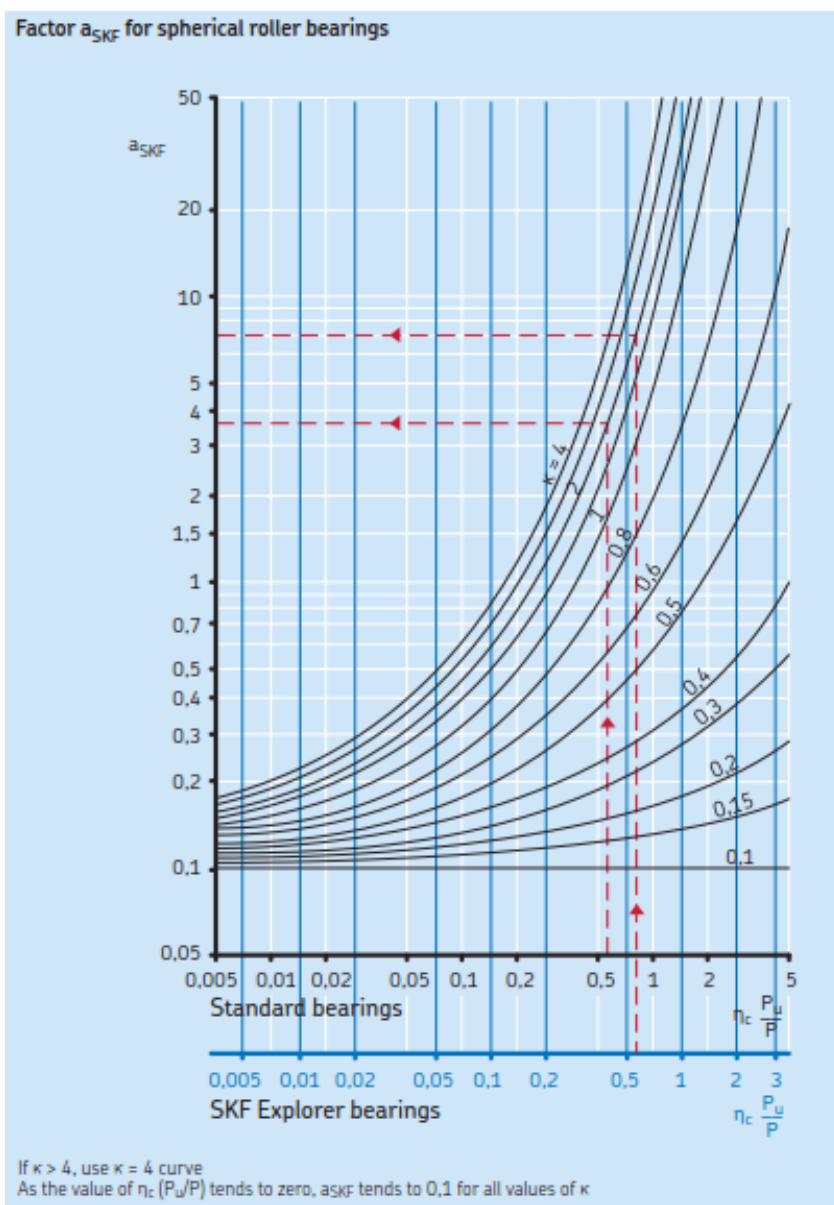

Figura 11. Fator $a_{s k f}$ para rolamentos autocompensadores de rolos.

Como o aço dos rolamentos SKF Explorer aprimorados é ainda mais resistente a danos superficiais devido à contaminação ou lubrificação deficiente que um rolamento SKF Explorer padrão, é importante considerar esta melhoria no cálculo de vida para estes rolamentos. Para tanto, os fatores de contaminação $\left(\eta_{c}\right)$ e viscosidade $(\mathrm{K})$ podem ser ajustados, conforme Figura 12:

\begin{tabular}{|c|c|c|}
\hline $\begin{array}{l}\text { Contamination } \\
\text { conditions }\end{array}$ & $\begin{array}{l}\text { Contamination } \\
\text { factor } \eta_{c}\end{array}$ & $\begin{array}{l}\text { Adjusted contamination } \\
\text { factor } \eta_{\mathrm{k} \cdot \mathrm{adj}}\end{array}$ \\
\hline $\begin{array}{l}\text { Typical contamination } \\
\text { Slight contamination } \\
\text { Normal cleanliness }\end{array}$ & $\begin{array}{l}0.2 \\
0.5 \\
0.7\end{array}$ & $\begin{array}{l}0,4 \\
0,6 \\
0,7\end{array}$ \\
\hline $\begin{array}{l}\text { Lubrication } \\
\text { conditions }\end{array}$ & $\begin{array}{l}\text { Viscosity ratio } \\
\kappa\end{array}$ & $\begin{array}{l}\text { Adjusted viscosity ratio } \\
\mathrm{K}_{\mathrm{sdj}}\end{array}$ \\
\hline Min. recommended film & $\begin{array}{l}0,4 \\
0,6 \\
0,8 \\
1\end{array}$ & $\begin{array}{l}0,5 \\
0,7 \\
0,9 \\
1\end{array}$ \\
\hline
\end{tabular}

Figura 12. Fatores de contaminação e viscosidade.

Para tornar mais fácil o cálculo com a influência desta melhoria, a Figura 13 fornece os fatores de ajuste finais que devem multiplicar o fator askf, já englobando os fatores $\eta_{c}$ e K: 


\begin{tabular}{|c|c|c|c|c|c|c|c|c|c|c|}
\hline Esti & ited rating & ife fact & for up & aded s & herical & Iller be & ings ar & CARB & sarings & \\
\hline & $\eta_{c} \quad 0,1$ & 0,2 & 0,3 & 0,4 & 0,5 & 0,6 & 0,7 & 0,8 & 0,9 & 1,0 \\
\hline k & & & & & & & & & & \\
\hline 4,0 & & $\begin{array}{l}2,0 \\
2.0\end{array}$ & $\begin{array}{l}1,3 \\
2,0\end{array}$ & $\begin{array}{l}1,0 \\
1,8\end{array}$ & $\begin{array}{l}1,0 \\
1,5\end{array}$ & $\begin{array}{l}1,0 \\
1,2\end{array}$ & 1.0 & 1,0 & 1,0 & 1,0 \\
\hline 3,0 & 1,0 & $\begin{array}{l}2,0 \\
2.0\end{array}$ & $\begin{array}{l}1.8 \\
2.0\end{array}$ & $\begin{array}{l}1,0 \\
1,7\end{array}$ & $\begin{array}{l}1,0 \\
1,5\end{array}$ & $\begin{array}{l}1,0 \\
1.2\end{array}$ & 1,0 & 1,0 & 1,0 & 1,0 \\
\hline 2,0 & 1,0 & $\begin{array}{l}2,0 \\
2,0\end{array}$ & $\begin{array}{l}2,0 \\
1.9\end{array}$ & $\begin{array}{l}1,4 \\
1,6\end{array}$ & $\begin{array}{l}1,0 \\
1,4\end{array}$ & $\begin{array}{l}1,0 \\
1,2\end{array}$ & 1,0 & 1,0 & 1,0 & 1,0 \\
\hline 1,0 & 1,0 & $\begin{array}{l}2,0 \\
1.9\end{array}$ & 2,0 & 2.0 & 1,6 & 1,3 & 1,0 & 1,0 & 1,0 & 1,0 \\
\hline 0,9 & 1,1 & 2,0 & 2,0 & $\begin{array}{l}1,9 \\
1,4\end{array}$ & 1,6 & 1,2 & 1,2 & 1,2 & 1,1 & 1,0 \\
\hline 0,8 & $\begin{array}{l}1,0 \\
1,2\end{array}$ & $\begin{array}{l}1,8 \\
2,0\end{array}$ & $\begin{array}{l}2,6 \\
2,0 \\
1,5\end{array}$ & $\begin{array}{l}1,4 \\
1,8 \\
1,\end{array}$ & $\begin{array}{l}1,3 \\
1,5\end{array}$ & $\begin{array}{l}1,1 \\
1.5\end{array}$ & $\begin{array}{l}1,1 \\
1,6 \\
13\end{array}$ & $\begin{array}{l}1, \frac{1}{1,7} \\
13\end{array}$ & $\begin{array}{l}1,1 \\
1,7\end{array}$ & $\begin{array}{l}1,1 \\
1,3\end{array}$ \\
\hline 0,7 & $\begin{array}{l}1,1 \\
1,2 \\
1,1\end{array}$ & $\begin{array}{l}1.7 \\
1.0\end{array}$ & $\begin{array}{l}1,0 \\
2,0 \\
1.4\end{array}$ & $\begin{array}{l}1,3 \\
1,6 \\
1,3\end{array}$ & $\begin{array}{l}1,2 \\
1,5 \\
1.2\end{array}$ & $\begin{array}{l}1,2 \\
1,6 \\
1,3\end{array}$ & $\begin{array}{l}1,3 \\
1,7 \\
1.3\end{array}$ & $\begin{array}{l}1,3 \\
1,8 \\
1,3\end{array}$ & $\begin{array}{l}1,3 \\
1,9 \\
1,4\end{array}$ & $\begin{array}{l}1,3 \\
1,0 \\
1,4\end{array}$ \\
\hline 0,6 & $\begin{array}{l}1,2 \\
1,1\end{array}$ & $\begin{array}{l}2,0 \\
1.5\end{array}$ & $\begin{array}{l}1,8 \\
1,4\end{array}$ & $\begin{array}{l}1,5 \\
1,3\end{array}$ & $\begin{array}{l}1.6 \\
1,3\end{array}$ & $\begin{array}{l}1.7 \\
1.3\end{array}$ & $\begin{array}{l}1,8 \\
1,4\end{array}$ & $\begin{array}{l}1,9 \\
1,4\end{array}$ & $\begin{array}{l}2,0 \\
1,4\end{array}$ & $\begin{array}{l}2,0 \\
1.4\end{array}$ \\
\hline 0,5 & $\begin{array}{l}1,3 \\
1,2\end{array}$ & $\begin{array}{l}1,9 \\
1.4\end{array}$ & $\begin{array}{l}1,6 \\
1,3\end{array}$ & $\begin{array}{l}1,6 \\
1,3\end{array}$ & $\begin{array}{l}1.8 \\
1.4\end{array}$ & $\begin{array}{l}1.9 \\
1.4\end{array}$ & $\begin{array}{l}2,0 \\
1,4\end{array}$ & $\begin{array}{l}2,0 \\
1.5\end{array}$ & $\begin{array}{l}2,0 \\
1.5\end{array}$ & $\begin{array}{l}2,0 \\
1.5\end{array}$ \\
\hline 0,4 & $\begin{array}{l}1,3 \\
1,2\end{array}$ & $\begin{array}{l}1,6 \\
1,3\end{array}$ & $\begin{array}{l}1,6 \\
1,3\end{array}$ & $\begin{array}{l}1,8 \\
1,4\end{array}$ & $\begin{array}{l}1.9 \\
1,4\end{array}$ & $\begin{array}{l}2.0 \\
1,5\end{array}$ & $\begin{array}{l}2,0 \\
1,5\end{array}$ & $\begin{array}{l}2,0 \\
1,6\end{array}$ & $\begin{array}{l}2,0 \\
1,6\end{array}$ & $\begin{array}{l}2,0 \\
1,6\end{array}$ \\
\hline 0,3 & 1,0 & $\begin{array}{l}1,3 \\
1,2\end{array}$ & $\begin{array}{l}1,2 \\
1,1\end{array}$ & $\begin{array}{l}1,2 \\
1,1\end{array}$ & $\begin{array}{l}1,1 \\
1,1\end{array}$ & 1,0 & 1,0 & 1,0 & 1,0 & 1,0 \\
\hline 0,2 & 1,0 & $\frac{1,1}{1.1}$ & $\begin{array}{l}1,1 \\
1,0\end{array}$ & $\begin{array}{l}1,1 \\
10\end{array}$ & 1,0 & 1,0 & 1,0 & 1,0 & 1,0 & 1,0 \\
\hline 0,1 & 1,0 & 1,0 & 1,0 & 1,0 & 1,0 & 1,0 & 1,0 & 1,0 & 1.0 & 1,0 \\
\hline
\end{tabular}

Figura 13. Fatores de ajuste de vida.

\section{CONCLUSÃO}

Este estudo apresentou as diferenças de desempenho e vida de rolamentos com microestrutura bainítica e rolamentos com microestrutura martensítica. Os 2 rolamentos com microestrutura bainítica, montados nos rolos longos (posição central), encontraram-se em boas condições visuais, sem sinais de fadiga. Já os 12 rolamentos com microestrutura martensítica, montados na mesma posição e que operaram durante a mesma campanha, encontram-se com fadiga subsuperficial em estágio avançado, além de trincas e fraturas por fadiga. Rolamentos autocompensadores de rolos são comumente utilizados em aplicações que requerem alto grau de confiabilidade, mesmo quando os níveis de contaminação e lubrificação deficiente são elevados. Para atender a estes requisitos, a SKF aprimorou os rolamentos de classe de desempenho Explorer, que já possuíam desempenho superior a um rolamento padrão. Combinando um aço limpo e homogêneo de alta qualidade com um processo de tratamento térmico diferenciado, a dureza do material foi elevada, sem que houvesse perda de tenacidade e aumentando a resistência ao desgaste. Esta evolução garante maior confiabilidade e aumento da vida em serviço, especialmente em aplicações cujas condições de operação são mais críticas, afetadas por contaminação ou lubrificação deficiente.

\section{REFERÊNCIAS}

1 Rolling bearings, PUB BU/P1 10000/2 EN, Agosto 2013.

2 ISO 15243:2004 - Rolling bearings - Damage and failures - Terms, characteristics and causes.

3 Bearing damage and failure analysis, PUB BU/I3 14219 EN, Março 2014.

$4 \quad$ SKF Explorer spherical roller bearings, PUB BU/P2 14346 EN, Dezembro 2013.

5 SKF Explorer self-aligning roller bearings, PUB BU/P9 11642/1 EN, Fevereiro 2012.

6 SKF Explorer self-aligning roller bearings upgraded for even longer service life, PUB BU/P8 12896/1 EN, Novembro 2013. 